\title{
Intelligent Health Monitoring Using Internet of Things
}

\author{
Kahkashan Tabassum* \\ Princess Nourah Bint Abdulrahman University, Saudi Arabia
}

*Corresponding author: Kahkashan Tabassum, Assistant Professor, Department of Computer Science, CCIS, Princess Nourah Bint Abdulrahman University, Saudi Arabia.

\section{Annotation}

Wireless medical sensor nodes are designed to include medical sensors to monitor human body conditions and store this information in memory. It will then be processed and analyzed. The sensors deployed on the human body mainly monitor body temperature, heartbeat, blood pressure, electrocardiogram and pulse oximetry. They send this information to a remote location for processing and a medical doctor can use these recorded readings to determine the condition of an individual or a patient. The data could be monitored on a regular basis that will help the doctor to be aware of the patients' status at specific instants of time [1].

The IoT has evolved as a smart ubiquitous framework of smart objects and is capable of hosting a diverse set of application realms such as business enterprises, health care, educational and social organizations, etc. This research is an attempt to utilize the IoT technologies to monitor health related issues in various health care centers, perform personal health monitoring of clinical patients, elderly patients care, chronic patients monitoring, clinical data collection regularly, children monitoring in order to keep them away from coming across insecure situations at home and patient monitoring in catastrophe situations. The research will also address current critical areas where growth of electrical simulators for accelerating bone development and electrical brain monitoring. These promote rehabilitation of patients with traumatic injuries using electrical simulators to accelerate bone growth. In addition, the research will use all the data generated from the monitoring phase to analyze, visualize and predict the various results. The analysis could be possibly used to study and understand the health status of patients living in the society so as to benefit them and help them to improve their health conditions and aid for building up of health-conscious attitude so that they realize the advantages of maintaining their fitness.

The idea of health monitoring using IoT needs a specific sensor which could be present or attached on various smart objects to connect to the large network of IoT. This connection will enable the sensor to send the essential medical data of the patients onto the remote objects spread within the area. The remote objects could be any smart electronic device that use medium of transfer of information as light waves or radio waves or motion energy.

Due to the use of IoT, the internet will transform medical systems allowing improved on spot and transitional patient care [2]. This research will use the framework of IoT to collect a patient's medical history and store it on internet or cloud server represented as medical records. Thus, the doctors can diagnose and suggest treatment for the patients either online or well before patient's arrival at the hospital. This research paper discusses about the strategies to attain integration of IoT based smart devices and services with smart healthcare services and will completely revolutionize the health care systems field.

Keywords: Internet of things; Electronic health records; Health informatics; Data Collection; Data visualization; Wireless medical sensor nodes

\section{Introduction}

Health informatics includes the health information systems, e-health services, electronic health records, clinical decision support systems, tele-health and many other established and emerging uses of information and communications technology in the health professions. The Health technology is assumed to be an application of structured knowledge, skills and expertise in the form of devices, medicines, vaccines, procedures and systems

developed to solve a health problem and improve quality of lives (World Health Organization).

Emerging technologies such as portable electronic devices (smart phones), social media, wikis, blogs, discussion forums and the huge cloud are offering support and services in different businesses as well as health fields. The future of health informatics and medical sciences is dependent on the essential's skills acquired 
by the professional practitioners based on the knowledge basics and professional attitudes. The dynamic changes in health Informatics and healthcare technology with its increased utilization has given rise to today's health care environment and the challenging responsibilities for doctors, nurses, the health information access and patients communication, diagnosis and cure of diseases including health promotion face many issues. It is evident that the upcoming technology improvements have opened new challenges for the consumers or patients of the health care system also. Hence the medical practitioners have to exercise a high sense of responsibility to work with patients and clients in the existence of this varying and navigating new systems and technology but still preserve patient perspective during their practice and effectively use the technology to assist the medical health condition of patients instead of manipulating the human condition to aid technology. There are various wireless network technologies that offer support in the health care and informatics field. This paper discusses the suitability of ubiquitous technologies to access medical health care information in the most effective manner.

Internet of Things (IoT) is an interconnection of several devices, networks, technologies and human resources to achieve a common goal. There is a variety of IoT based applications that are being used in different sectors and have succeeded in providing huge benefits to the users. The integration of various technologies like ubiquitous technology together with IoT is reducing the communication gap that is existing between technologies and giving rise to highly potential environment to support accessibility of resources in the enterprises and hospitals. This integration offers significant benefits in terms of minimized deployment costs and providing secure precaution to patients.

The research for best support in terms of wireless technologies for medical device communications in various healthcare environments states the fact that there is a wide range of potential applications and use case scenarios which can be applied on patient's hospital bedside to a doctor's prescription. Medical applications are real time and are based on severe requirements of accuracy or latency Some of these include alarm notifications, waveform delivery, e-prescription, etc. which cannot afford to lose or delay data since it has a life and death implication. Thus, the wireless medical sensors carrying these applications must be deployed on a patient's body or patient's bedside with extreme care and safety. Even the hospital databases and Internet access completely depend on entirely flawless deployment of network connecting different hospitals and rely on high-speed bandwidth links for medical applications in use. All the wireless medical sensors have the minimum requirements of consistent communication, energy efficient, mobile and multicast support to prompt accurate delivery of data.

A sensor node comprises of wireless medical sensors to monitor human body conditions and they sense human body temperature, heartbeat, blood pressure, electrocardiogram and pulse oximetry and send this information to a remote location for processing and a medical doctor can use these recorded readings to determine the condition of an individual or a patient. Later it can be processed and analyzed to diagnose various medical conditions [3]. The sensors deployed on the human body mainly monitor the data on a regular basis, had the doctor found any abnormality with the patient condition he could quickly arrange for required treatment. This method benefits many patients and keep them away with paying regular visits to a doctor and only approach a doctor in emergency only. The sensors implanted in the human body are portable, wearable and adjustable [4]. Medical health monitoring includes monitoring clinical issues of patients, chronic patients monitoring, care and monitoring of old-aged, patient emergency and child health monitoring. In this paper we discuss how the assessment and visualization of data by monitoring their medical health using IoT.

The Internet of Things (IoT) is mega-technology that is expected to establish connection with all any-s (anything-anyone-anytimeanyplace-any service-any network). It influences the whole block chain of businesses and have smart objects and devices, systems and services that identifies a unique heterogeneous network connectivity [5,6]. (Figure 1) shows an IoT based health care network that illustrates how the evolution of technology in medicine will look in practice. A patient ID card, when scanned, connects to a protected cloud which stores the electronic health record (EHR) vitals of medical histories of patients. Doctors and nurses can access EHR on any ubiquitous device or desktop computer.

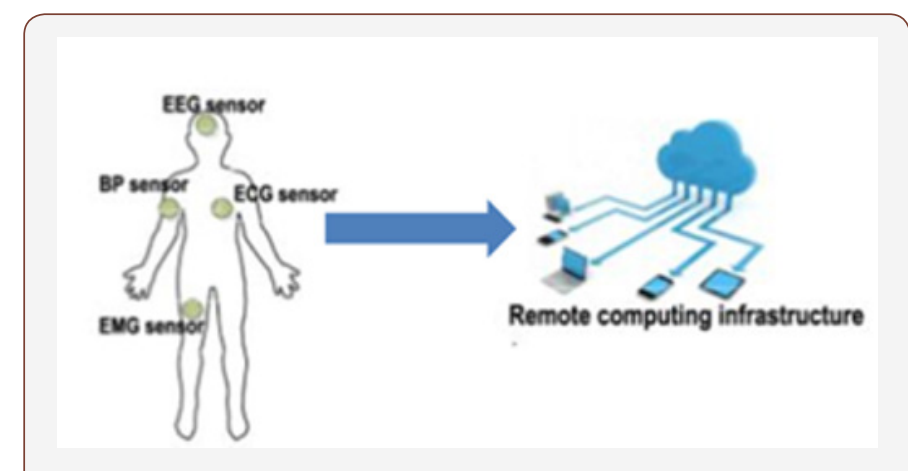

Figure 1: An example - IoT based healthcare network.

In recent days many people have the provision of wearables and mobile apps for collaborative nt fitness and health support and management. Also, these devices are used by patients for symptom tracking and care coordination. All these platform analytics can advance the appropriate data interpretations at the same time simplifying the amount of time to benefit end users. The Insights extended from big data analysis can motivate for the digital snooping of the healthcare world, business processes and real-time decision-making. [7] and encourage a new category called Digital Health Advisors will arise. These Advisors possess the skills and are capable of interpreting and understanding health and safety of data. These will become increasingly important to the global population. The Limitations of using IoT has to be further identified and could be studied to provides a security model with the aim to minimize security risk in IoT and eHealth fields. The next section provides the objectives of the proposed research.

\section{Objectives}

The aim of the research work is to accomplish the following objectives: 
a) To implement new biomedical personal wireless networks monitoring different body signals related to different diseases, treatment, and the patient treatment life cycle.

b) Monitoring for Chronic and old aged patients living in an elderly care center.

c) To create Patient Emergency Monitoring and tracking system.

d) To create a child monitoring system that ensures child protection and safety

e) To create and maintain long term databases on the cloud.

f) Improve monitoring consistency and enhance data quality and precision for decision support leading to better therapeutic interventions.

g) The data generated by applying the patient monitoring techniques can be used to analyze, visualize, predict and find finally solutions to the unsolved problems in the healthcare system.

h) The results obtained through analysis and visualization could be used to infer and decide the condition of a patient's health and provide them with suitable relief procedures or solutions.

i) To help the society by increasing awareness about the various solutions and treatments to the diseases among the patients and also providing individuals with the knowledge from protection and prevention from these diseases.

\section{Background and Related Work}

The IoT supports a wide range of applications including smart cities, traffic congestion, waste management, structural health, security, emergency services, logistics, retails, industrial control, and health care [8-11]. Medical informatics field is keen towards technological prospects to apply the latest techniques in the field to enhance medical education including medical practice and research $[12,13]$ through the developments in information processing and communication technology focusing on medical application development rather than patient's perspective. Greenes and Shortliffe wrote: "After many years of development of information systems to support the infrastructure of medicine, greater focus on the needs of physicians and other health care managers and professionals is occurring-to support education, decision making, communication, and many other aspects of professional activity [14]. The classic medical informatics textbooks seldom have chapters on the information requirements of consumers [15]. Consumer Health informatics analyzes consumers' needs for information, related studies and implements strategies making the information available to consumers. CHI integrates consumers desires into medical information systems. Consumer informatics is most challenging field and it involves many different disciplines including public health, nursing, health promotion, health education, library and communication [16,17].

Electronic Health records [18] are used to make health information available to patients or consumers. Computer aided decision making is helping the patients to make their choice appropriately. Many programs from the internet are used to educate patients or consumers $[19,20]$. Wireless Sensor Nodes are designed to include medical sensors to monitor human body conditions and they sense the required data and stores in memory which will be processed and analyzed to evaluate various medical situations and any disorders or deviations from normal rate perceived within a human body. The sensors deployed on the human body mainly monitor body temperature, heartbeat, blood pressure, pulse oximetry, etc. and they send this information to a remote location for processing and a medical doctor can use these recorded readings to determine the condition of an individual or a patient. The data could be monitored on a regular basis that will help the doctor to be aware of the patients' status at every instant of time. Had the doctor found any situation with the patient condition he could quickly call for the treatment. Early treatments are the best solutions in medical health cases. This method avoids the patients to wait and pay regular visits to the medical doctor instead they can stay at home and whenever a specific condition arises that may lead to serious consequence then only, they can meet the doctor. Like WSNs, Wireless Medical Sensor Nodes (WMSNs) are based on underlying wireless and ubiquitous networks but are more promising when compared to the previous. WMSNs offer quality healthcare applications since they can be deployed in many ways on human body. They are portable, wearable, adjustable and can be implanted in the human body.

Disaster scenarios demands extremely efficient, scalable and cost-preserving elements for observing and monitoring patients. These may utilize paper triage or mi-Tags that process patient data, patient monitoring software, and USB receiver [21]. Due to limitations of wireless networking only limited number of patients would be accommodated per network as given in Nihon Kohen's solution [16]. The existing medical monitors are costly with poor integration of workflows. Few commercial embedded sensors can be found in garments for example MagIC, Sensatex and Life Shirt [22]. Some academic research bases like ACT at the University of Alabama and Bodynets at UCLA [15], uses body area network of sensors to detect and enroute sensor data to the receiver through Wi-Fi supported PDA [7]. To implement Health Monitoring using Internet of Things that would be useful to the patients in the emergency situations such as disaster or surge scenario for reaching hospitals and accessing the ambulances [23]. Since as a result of disaster, a chaos is found that destroys the resources and hence the communication suffers, and its infrastructure is damaged leading to unreliable environment [23]. But all these projects suffer from few limitations in case of casualty's scenarios.

Medical care and health care are the most fascinating and developing application areas for the IoT [6,24]. IoT offers incredible prospective rise to huge number of medical applications in the 21st century like fitness care and development programs, chronic diseases and remote health monitoring, old aged patient monitoring and much more. The IoT is developed as a smart pervasive framework of smart devices. There exist different technologies to reduce the costs to manage the patient's sickness. Some patients are also using mobile apps interfaced with tele health and telemedicine 
by means of medical Internet of Things (mIoT) [18]. The mIoT and big data in healthcare fields is paving ways for effective prevention and treatment methods for relieving the patients $[25,26]$.

\section{Research Project Design \& Methodology}

This section explains the manner in the research project will be operated and the research techniques it uses - the specific means, strategies, approaches and tool that would be used to gather/collect data, how the data will be analyzed along with the inference's creation.

\section{Literature review}

The research project has carried out the related survey and similar systems study and hence it is expected to enable to create a novel working model based on the existing models studied.

\section{Analysis}

In this we determine whether the research being pursued is feasible. We make feasibility report for functional and nonfunctional requirement. Various analysis tools like data flow diagram, flowchart, entity-relationship diagrams, UML diagrams will be used to understand the structure of the research model.

\section{Designing model}

Will create a working model through which testing of results will be done. We use the hardware and software tools mentioned in the next section (Implementation).

\section{Experimental system}

The working model will be used to tabulate the data which is received by testing and experimentation. Steps to perform experimentation. A method of Experimental computer science and engineering (ECSE) will be used to the building of experimentation on hardware and software systems [27]. In ECSE experimental evaluation is studied first and later it will be focused on the artifacts that have been created by an individual. The Artifacts we use in the experimental system are the computers, chips, compilers, editors, microcontrollers, ADC, expert systems, protocols, etc. These will be used to make direct analysis of the problem and determine how the formulated computational idea would perform on the logical analysis.

\section{Analysis and visualization}

The final step is to analyze, visualize and predict the effect on an individual (here patient) and further assist them to improve their health statistics. High volume of raw data will be gathered from various platforms (experimental set-up) having sources or devices and requires parallel computing for loading the data, preprocessing, and analyzing it. [Dell EMC]. Different techniques (as specified in Management Plan) will be used to deal with the data and to extract knowledge and useful information and to assist in decision making. Big data in the health care industry can help reduce cost of care and provide better quality of care. It can also prevent diseases. The lifecycle of data analysis:

a) Understand the problem, specify the required resources, and define the hypothesis. b) Understand the data and prepare it for analysis by applying preprocessing techniques such as cleaning the data from noise and filling missing values.

c) Understand the relationship between the variables, decide which are the most Important variables, and plan what model to use.

d) Build the selected models using the training sets.

e) Test the performance of the models using the test sets to examine how successful the models are. If the results are successful, they have to be introduced in a useful way.

f) Operationalize once the model is ready for production.

\section{Writing paper in a journal}

The academic achievement will be written, and it shares the magnitude and significance of ideas, impacts and results. The contribution will be used directly to help other to understand better, the topic or a question. It may even indicate the impossibility of certain goals of research and so on. The quality of the journal/ conferences that published the result will be with high impact factor.

\section{Experimental Implementation}

The following section discusses the details of hardware and software requirements to create the platform for Data Collection for the research project.

\section{Data collection}

What data will you collect or create? The research involves sensors, internet and server support. The sensors will be deployed on the human body (patients) and are responsible to record the data of specific essential elements of human body such as heart beat, blood pressure, body temperature, ecg, etc. Although the data is in analog form it is converted by a sensor node to its corresponding digital form by means of the ADC (Analog to Digital Converter) found within the sensor node. Since the data will be collected regularly it will be huge and is stored on day -to -day basis in the server or it could be selected at regular intervals for monitoring old-aged people or children or it may be storage in emergency situations. In all the cases mentioned the data collected is more and has critical value. The data could be uploaded to the cloud as well provided we connect and use one. In this research we assume the data to get stored on server (medical) or project-server we install/ connect to store the data sent from the sensor into it, over the internet. It is anticipated to generate 20-50 KB of data for one upload (every 2 hourly) from each case mentioned except the emergency monitoring case. So, one can imagine the weekly data generated and finally monthly to yearly data its huge, its big data. The effectiveness of research results is based on the amount of data collected. Also, the tools which will be used to analyze the data such as Knime, open refine, PubMed, orange, R programming, datawrapper, nodeXL, Weka or SPSS. The task of data collection will be shared among the members and every member will be assigned to a specific data station. There are 5 data stations (dat_stn1, dat_stn2, dat_stn3, dat_stn4 and dat_stn5 - refer to objectives of research) corresponding to the five different cases of monitoring 
mentioned in the research and corresponding to the members in the research. To read this data from sensor we use PHP code. Through coding we can versionalize the data, calibrate it, repeat samples or measurements and standardize data recording or capturing.

How will the data be collected or created? There are tools like Raspberry Pi data online that will be used to store data online after receiving. These tools can be programmed to store the data in a tabular formed to with the columns as ID no assigned to each sensor, Date-Time column and the columns corresponding to the sensors recording the essential medical parameters on patients' body. A reasonable range for this sensor kit with one sensor would be around $100 \$$ and needs to be interfaced with a Webserver which may be @10\$ per month. All the files would be stored under a new folder of our choice (data source/database/table - iot_data. sql or an extension corresponding to the support tool selected). If you use free host namely bluehost website, we can create a folder called pnu_hm_iot_01.tv for website. Then the files corresponding to 5 stations will be created and populated with the data. Different files will be created per day or per week to keep the amount of data uniform within a file source. The program will use PHP and My SQL on server side. The program will insert values of the sensor data received in the database by reading the data from sensor. To read this data from sensor we use PHP code. High volume of raw data will be gathered from various platforms (experimental setup) having sources or devices and requires parallel computing for loading the data, preprocessing, and analyzing it [Dell EMC]. Different techniques (as specified in first paragraph) will be used to deal with the data and to extract knowledge and useful information and to assist in decision making. Big data analysis in the health care industry can help reduce cost of care and provide better quality of care in addition too preventing suffering and diseases in patients in a smart way.

Steps for implementation platform for smart monitoring using IoT: In this section the experimental set up using raspberry pi platform is explained.

a. Installation of Raspberry Pi OS. To download the OS, go to www.raspberrypi.org website. (Figure 2)

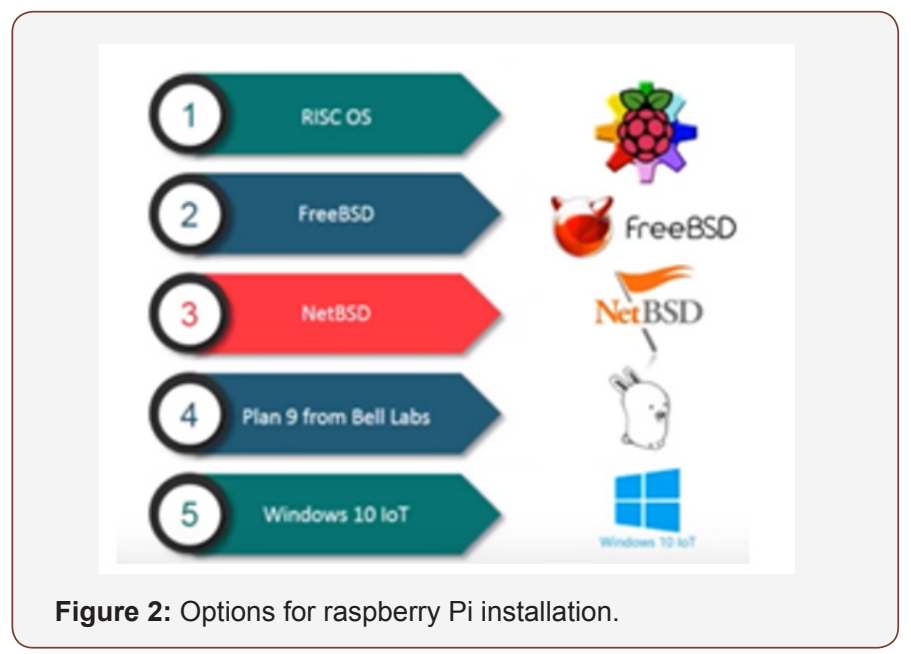

b. Download Noobs and ensure the use of 32GB memory card since minimum memory requirement for NOOBs is $16.5 \mathrm{~GB}$. (Figure 3)

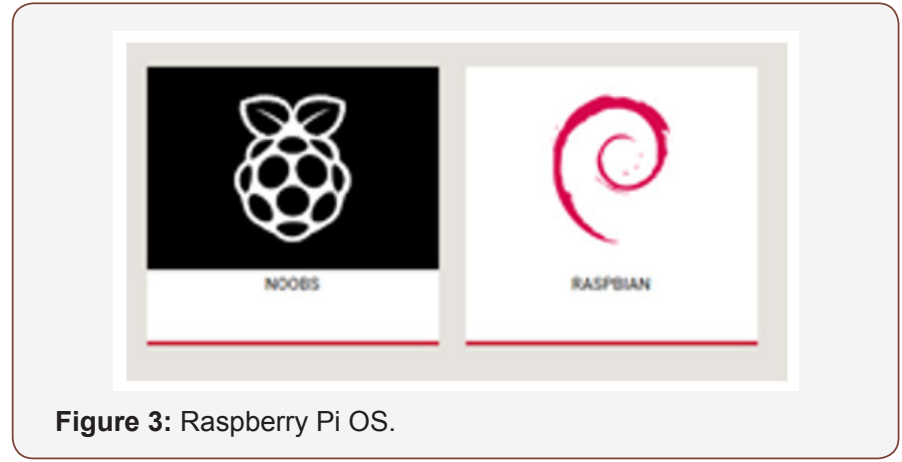

c. Extract the files from the NOOBs folder.

d. Copy these files to a formatted SD card.

e. Now put these files in the Raspberry Pi SD Card Slot at the bottom of it.

f. Follow through the instructions further for installation. Now Rasbian OS is installed in the Raspberry Pi. Figure 4 illustrates the implementation setup for the IoT when it is ready to collect data and the required interfacing with the required medical sensors for the regular data gathering and storing in internet(web browser) or cloud. The following steps are applied further to collect data from the medical sensors deployed on the raspberry Pi.

(1) Deploy the corresponding medical sensors on human body.

(2) Use Raspberry Pi to transfer data and display results on the sensor board.

(3) Use Instapush API and Python Script to push these notifications across the internet (Figure 4).

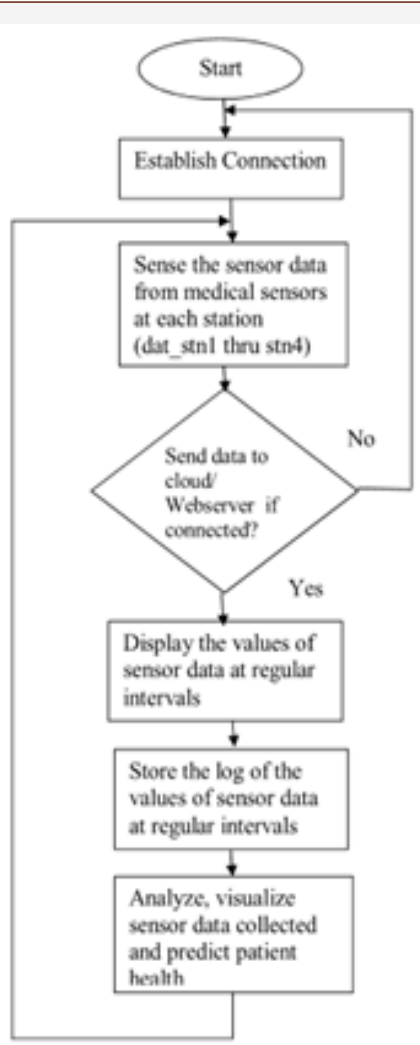

Figure 4: Flowchart for implementation activities. 
Documentation and meta data: What documentation and metadata will accompany the data? The activities and phases will be documented to include all the details about implementation both at client and server side. This documentation can be used by the future projects to improve the existing system. In addition, it will include the names of all people who worked for its development. In other words, we will generate a research report. The work will be accompanied by regular and weekly presentation and compilation of weekly progress will be recorded to create the final documentation. The research will be published in SCOPUS/ISI journal.

Storage and backup: How will the data be stored and backed up during the research? The data has to be recorded regularly on a Webserver. Before reaching the webserver, it needs an intermediate storage and also a backup storage. The research needs sensor with memory devices, microcontrollers, analog to digital convertor to create a sensor node, database server, backup server and webserver space. All these needs to be purchased. The data will be regularly backed up so in case of any incident we can use its backup. How will you manage access and security? The research will have to address the security issues and control the data access. Since the research uses sensitive data about human medical data (electronic health record) it is needed to ensure security. During the research work while collecting the data, the collaborators will seek permission to access the data. The research project will use appropriate security measures and also ensure security while the moving the data.

Selection and Preservation: Which data are of long-term value and should be retained, shared, and/or preserved? The data generated throughout the research has to be preserved since the data collected will be used to analyze, visualize and predict the medical status of patients and likewise improve their health and provide the relevant cure or treatment. What is the long-term preservation plan for the dataset? The data will be stored in the university research unit so that it can be further used. The data will be archived in the webserver and the database server provided the costs of maintenance are taken care off by the research unit.

\section{Conclusion}

The IoT is a potential integrated network with huge impact on medical field. It enables smart devices to collect and exchange data. The convergence of medical and information technologies will reform these areas by minimizing their costs and inefficiencies. To use the modern innovative technologies in a secure healthcare context, the study and establishment of eHealth policies and regulations is important. The results utilization is as follows:

a) It will be useful and benefit both the private and public medical and health centers.

b) It will support fitness and health education areas.

c) It will of offer collaborative prevention and treatment of patients by prediction of various medical issues and sickness of patients.

d) The computational intelligence techniques implemented will be used to analyze, visualize, predict and find finally solutions to the unsolved problems in the healthcare system. e) It analyzes the data collected by sensors to infer and decide the condition of a patient's health and relief.

f) It will provide smart platform for smart objects and smart apps.

g) Using the big data generated, it addresses various IoT and eHealth for future research across the world to determine how they can facilitate economies and societies in terms of sustainable development;

h) It is anticipated to help in future and advanced research in the areas of mIoT

i) It opens new avenues for future research on IoT-based health care systems with open issues and challenges.

\section{Acknowledgements}

It gives an immense pleasure to submit this research paper on "Intelligent Health Monitoring using Internet of Things (IoT)". I am thankful to most respected members of the Computer Sciences Department research unit for their constant encouragement and support in inspiring to do the research projects to enhance the research skills of the women researchers of Princess Nourah University. I would also like to express a deep sense of gratitude to my co-researchers without whom this research could not have its inception and progress. I am very much thankful to the Dean and Head of department of Computer Science for their persistent motivation and inspiration. I would like to extend my heartfelt thanks to Dr Hadil Shaiba for spending her valuable time in providing many helpful suggestions.

\section{Conflict of Interest}

No Conflict of Interest.

\section{References}

1. Kahkashan Tabassum, A Damodaram, SVS Rama Krishnam Raju (2013) An Energy-Efficient New Hierarchical Stable Election Protocol for Wireless Sensor Networks. Proceedings of $2^{\text {nd }}$ International Conference on Recent Advances in Design, Development and Control of Micro-AirVehicles, IC-RA-MAV.

2. Minaie Afsaneh, Reza Sanati-Mehrizy, Paymon Sanati-Mehrizy, Reza Sanati-Mehrizy (2012) Integration of Wireless Sensor Networks in the Computer Science and Engineering Curricula. Proceedings of ASEE Annual Conference.

3. Sghaier Nouha, Abdelhamid Mellouk, Brice Augustin, Yacine Amirat, Jean Marty, et al. (2011) Wireless Sensor Networks for Medical Care Services. In 7th International Wireless Communications and Mobile Computing, IEEE

4. K Natarajan, B Prasath, P Kokila (2016) Smart Health Care System Using Internet of Things. Journal of Network Communications and Emerging Technologies (JNCET) 6(3): p 10.

5. J Höller, V Tsiatsis, C Mulligan, S Karnouskos, S Avesand, (2014) From Machine-to-Machine to the Internet of Things: Introduction to a New Age of Intelligence. Amsterdam, Elsevier, The Netherlands.

6. Ali Calhan, Sedat Atmaca (2013) A new Network Coordinator Node Design Selecting the Optimum Wireless Technology for Wireless Body Area Networks. KSII Transactions on Internet and Information Systems, 7(5): 1077-1093.

7. L Yang, Y Ge, W Li, W Rao, W Shen, et al. (2014) A home mobile healthcare system for wheelchair users. IEEE Int Conf Comput Supported Cooperat, Work Design (CSCWD) pp. 609-614. 
8. Mirza Mansoor Baig, Martin J, Hamid Gholam (2013) A comprehensive survey of wearable and wireless ECG monitoring systems for older adults. Med Biol Eng Comput 51(5): 485-495.

9. Kahkashan Tabassum (2018) An Intelligent and Robust Strategy for Patient Monitoring to Provide Emergency Services. SciFed J Com Science.

10. C tang, Yu Dong, Jain Ye, Ming Zhang (2014) Research and development of self-organized protocol in wireless body area network.

11. Cuneyt Bayilmis, M Younis (2010) Energy-aware Gateway Selection for Increasing the Lifetime of Wireless Body Area Sensor Networks. J Med Syst. 36(3): 1593-1601.

12. VM Rohokale, NR Prasad, R Prasad (2011) A cooperative Internet of Things (IoT) for rural healthcare monitoring and control. pp. 1-6.

13. SM Riazul Islam, Daehan Kwak, MD Humaun Kabir, Mahmud Hossain, Kyung-Sup Kwak, et al. (2015) The Internet of Things for Health Care: A Comprehensive Survey. IEEE Access Journal.

14. Ismail Kirbas, (2012) Health Face: A web-based remote monitoring interface for medical healthcare systems based on a wireless body area sensor network. Turk J Elec Eng \& Comp Sci, 20(4): 629-638.

15. Dishongh Terrance, Michael Mc Grath (2009) Wireless Sensor Networks for Healthcare Applications. Artech House Publishing.

16. Y J Fan, Y H Yin, LD Xu, Y Zeng, F Wu (2014) IoT-based smart rehabilitation system. IEEE Trans. Ind. Information, 10(2): 1568-1577.

17. Ashraf Darwish, Aboul Ella Hassanien (2011) Wearable and Implantable Wireless Sensor Network Solutions for Healthcare Monitoring. Sensors (Basel), 11(6): 5561-5595.

18. Bertocco M, Gamba G, Sona A, Vitturi S (2007) Performance measurements of CSMAICA-based wireless sensor networks for industrial applications. Instrumentation and Measurement Technology Conference Proceedings, IMTC, IEEE.
19. Kahkashan Tabassum, A Damodaram (2011) A Multicast-Based Data Dissemination to Maintain Cache Consistency in Mobile Environment. Communications in Computer and Information Sciences, (132): 290301.

20. SmartDust, University of California, Berkeley. Departments of Electrical Engineering and Computer Sciences, Mechanical Engineering, and Bioengineering of UC Berkeley, and Department of Surgery of UC San Francisco.

21. Kumar Pardeep, Lee SG, Lee HJ (2012) E-SAP: Efficient-Strong Authentication Protocol for Healthcare Applications Using Wireless Medical Sensor Networks. Sensors, 12(2): 1625-1647.

22. Ko JG, Lu C, Srivastava MB, Stankovic JA, Terzis A, et al. (2010) Wireless Sensor Networks for Healthcare. Proceedings of the IEEE.

23. Diksha B Wasankar, Vijay S Gulhane, LK Gautam (2017) Application of Internet of Things in the Field of Medical and Smart Health Care. International Journal of Innovative Research in Computer and Communication Engineering.

24. Kaleem Ullah, Munam Ali Shah, Sijing Zhang (2016) Effective Ways to Use Internet of Things in the Field of Medical and Smart Health Care. IEEE.

25. Pang Z (2013) Technologies and architectures of the Internet-of-Things (IoT) for health and well-being.

26. Emeka E, Fapojuwo A (2011) A Survey of System Architecture Requirements for Health Care-Based Wireless Sensor Networks. Sensors, (5): 4875 - 4898.

27. Akshay Gapchup, AnkitWani, Durvesh Gapchup, Shashan K Jadhav (2016) Health Care Systems Using Internet of Things. International Journal of Innovative Research in Computer and Communication Engineering 4(12). 